\title{
Entropy Production in Heavy-Ion Collisions
}

\author{
C. Das \\ Institute of Physics, Sachivalaya Marg, Bhubaneswar 751 005, India
}

\author{
R. K. Tripathi
}

Institute of Physics, Sachivalaya Marg, Bhubaneswar 751 005, India, and Physique Nucléaire Théorique, Institut de Physique au Sart Tilman, Université de Liège, B-4000 Liège 1, Belgium

and

\section{J. Cugnon}

Physique Nucléaire Théorique, Institut de Physique au Sart Tilman, Université de Liège, B-4000 Liège 1, Belgium (Received 28 January 1986)

It is shown that a fully self-consistent equation of state with realistic interactions gives entropy in excellent agreement with recent experimental data. We suggest that entropy production can be reasonably approximated by a free Fermi gas with effective mass.

PACS numbers: $25.70 .-\mathrm{z}, 21.65 .+\mathrm{f}$

One of the main motivations of heavy-ion physics is to study the bulk properties of baryonic matter under unusual conditions of densities and temperatures. Although the information about the properties of the dense hot matter thus created is somewhat obscured by the fact that the matter remains hot and dense only for a very short time $\left(<10^{-22} \mathrm{sec}\right)$, thus raising many fundamental questions including thermalization, it has been proposed ${ }^{1}$ to look into the entropy production as one of the observables providing information on such a matter. This generated a lot of activity ${ }^{2-11}$ in the area, including the search for phase transitions to exotic matter. The general feeling seems to be emerging to stay still within the baryonic matter. The situation has taken new dimensions in the light of new experimental observations. $^{2}$

These experiments have been done for $E_{L}=42,92$, and $137 \mathrm{MeV} /$ nucleon and correspond to average temperatures $T_{\mathrm{Av}}=18,25$, and $35 \mathrm{MeV}$, temperatures above the critical point, and thus provide information about the stable matter beyond the region of instability. The corresponding density of matter, another piece of information needed to specify the entropy, lies in the range of $(0.3-0.7) \rho_{0}$; however, the best fit is found with $\rho=0.5 \rho_{0}$. These experiments have galvanized the activity in the area. The present Letter provides an alternative explanation of the experimental observations within a fully self-consistent model using realistic $N N$ interactions and gives a coherent picture of the entropy production in heavy-ion collisions without assuming the breakup of the phases.

The basic ingredient in studying the bulk properties of matter is its equation of state. Consequently it is vital that a reliable fully self-consistent equation of state with realistic interactions must be available before any definite conclusions can be drawn. Calculation of the equation of state involving realistic interactions among other things should accurately account for the strong nature of the interaction. It should also properly account for the scattering to intermediate states. In addition the degeneracy and the single-particle potential should be calculated self-consistently as well. The formalism presented here takes into account all these things self-consistently and consequently gives the fully self-consistent realistic equation of state.

At zero temperature, Brueckner theory is commonly used to account for the strong nature of the interaction in terms of two-body potentials. However, because of compression, the matter in heavy-ion collisions gets heated up. One should, therefore, bring in the temperature effects in the formalism as well.

In the finite-temperature case ${ }^{12}$ one starts by calculating the grand thermodynamic potential $(\Omega)$ per unit volume:

$$
\Omega=-p=-T \ln \operatorname{Tr} \exp [-(H-\mu n) / T],
$$

where $H, p, T, \mu$, and $n$ are the Hamiltonian, pressure, temperature, chemical potential, and number density, respectively. The thermodynamic potential can be expanded as a linked-cluster expansion, i.e.,

$$
\Omega=\Omega_{0}+\Omega_{1}+\Omega_{2}+\ldots,
$$

where $\Omega_{0}, \Omega_{1}, \Omega_{2}, \ldots$ are the contributions to the thermodynamic potential due to the unperturbed part, one-body part (single-particle potential), and two-body part (binary collision) of the Hamiltonian. It must be noticed that our formalism is limited to $\Omega_{2}$, but using the Brueckner reaction matrix instead of the bare $N N$ force. The number density $n$ is given by

$$
n=\sum_{\tau} n_{\tau}=\sum_{\tau}\left\{1+\exp \left(\left[\epsilon_{\tau}(k)-\mu_{\tau}\right] / T\right)\right\}^{-1},
$$

where $\mu_{\tau}$ and $n_{\tau}$ are the chemical potential and number density of nucleons with isospin $\tau$ ( + for pro- 
tons and - for neutrons). Here we have put the Boltzmann constant equal to 1 . The proton-to-neutron ratio is defined by $r=n_{+} / n_{-}$. The single-particle energy is given by

$$
\boldsymbol{\epsilon}_{\tau}=(\hbar k)^{2} / 2 m_{\tau}+U_{\tau}(k) \text {, }
$$

where $U_{\tau}(k)$ is the single-particle potential and has to be determined self-consistently from the equation ${ }^{11,13}$

$$
U_{+}\left(k_{1}\right)=\left(1 / 4 \pi^{2}\right) \int_{0}^{\infty} d k_{2}\left[n_{+}\left(k_{2}\right) g_{++}\left(E_{S}, k_{1}, k_{2}\right)+n_{-}\left(k_{2}\right) g_{-+}\left(E_{S}, k_{1}, k_{2}\right)\right] \text {, }
$$

where the $n$ 's in the above equation are the Fermi distribution functions defined in Eq. (3) and the $g$ 's are the interaction matrices [Eq. (6)]. It is important to emphasize the following concerning the single-particle potential. One notes that a single-particle potential is needed in $n\left(k_{2}\right)$ and $g$ in order to calculate the singleparticle potential itself. Consequently one must calculate it by iteration. Besides, the chemical potential $\mu_{\tau}$ [Eq. (3)] has to be determined self-consistently as well. For a given density and temperature at each point both of these self-consistencies have to be achieved. In addition, the reaction matrix [Eq. (7)] has to be calculated at these self-consistent values as well. Consequently the whole procedure is rather involved and tedious and a lot of vigilance has to be exercised.

The $g$ 's are (e.g.)

$$
g_{\tau \tau}\left(E_{S}, k_{1}, k_{2}\right)=\frac{\tan ^{-1}\left[\pi \rho_{E} Q_{\tau \tau} K_{\tau \tau}\left(E_{S}\right)\right]}{\pi \rho_{E} Q},
$$

where $\rho_{E}$ is the single-particle level density and the $K$ matrix satisfies the integral equation

$$
K_{\tau \tau}\left(E_{S}\right)=V_{\tau \tau}+V_{\tau \tau}\left[Q_{\tau \tau} /\left(E_{S}-H_{0}\right)\right] K_{\tau \tau},
$$

where $V_{\tau \tau}$ is the realistic nuclear interaction and $Q_{\tau \tau}$ is

the Pauli operator

$$
Q_{\tau \tau}=\left[1-n_{\tau}\left(k_{1}\right)\right]\left[1-n_{\tau}\left(k_{2}\right)\right],
$$

and $E_{S}$ is the starting energy of the two particles and is given by

$$
E_{S}=\left(\hbar^{2} / 2 m_{\tau}\right)\left(k_{1}^{2}+k_{2}^{2}\right)+U_{\tau}\left(k_{1}\right)+U_{\tau}\left(k_{2}\right) .
$$

It should be emphasized that a lot of care must be taken in calculating $K$ with the above prescription of $Q$. We do not go into the intricacies of this here. This needs a detailed discussion and will be reported elsewhere. $^{14}$

The equation of state is then calculated by

$$
P=-\pi^{-2} \int_{0}^{\infty} d k k^{2} n_{\tau}(k)\left[\frac{1}{3} k d \epsilon_{\tau} / d k+\frac{1}{2} U_{\tau}(k)\right] .
$$

For our purpose we have chosen the Sussex interaction, ${ }^{15}$ which is derived directly from the experimental phase shifts and has been adjusted to give the simultaneous saturation of nuclear matter and finite nuclei. Other realistic interactions derived from the phase shifts would not alter the conclusions discussed here.

The entropy has been calculated by the formula

$$
S=-n^{-1} \sum_{\tau} 2(2 \pi)^{-3} \int d^{3} k\left\{n_{\tau}(k) \ln n_{\tau}(k)+\left[1-n_{\tau}(k)\right] \ln \left[1-n_{\tau}(k)\right]\right\} .
$$

Our equation of state gives $17.3 \mathrm{MeV}$ (for $r=1$ ) for the critical temperature of the first-order liquid-vapor phase transition. The importance of this result is that above this temperature, which is what we are discussing here, surface, shape, and other specific finite-size effects are less important and bulk properties of matter largely dominate. Figure 1 shows the entropy per baryon as a function of density for different temperatures. The experimental results of Ref. 2 are shown by the shaded areas. The horizontal, inclined, and vertical lines are for $T=18,25$, and $35 \mathrm{MeV}$, respectively. It is gratifying to note that our results are in good agreement with experiment. The agreement would be even better if (a) the freezeout density increases with beam energy as is usually expected and (b) pions and $\Delta$ resonances (which begin to show up for temperatures $\sim 30 \mathrm{MeV})$ were taken into account in the calculation as well, since they have been accounted for in the experimental analysis.

It should be noted, however, that the agreement rests on the assumption that the quoted temperatures can be understood as true temperatures, i.e., as representing the chaotic nucleon motion at the freezeout. Taking account of a possible global expansion would probably decrease the temperatures.

It should be emphasized that for very low densities $\left(<0.025 \mathrm{fm}^{-3}\right)$ it is very important to determine the density very accurately through experimental analysis before making any meaningful comparison with theoretical results since the entropy curves are very steep at these densities.

Our results are in complete agreement with the low-temperature calculations of Friedman and Pandharipande, ${ }^{16}$ giving us full confidence in our method, particularly in view of the fact that two entirely different methods with realistic interactions converge at the same entropy at low temperatures. This statement is substantiated and enlightened by Fig. 1, where the dashed lines are the results for the free Fermi gas with 


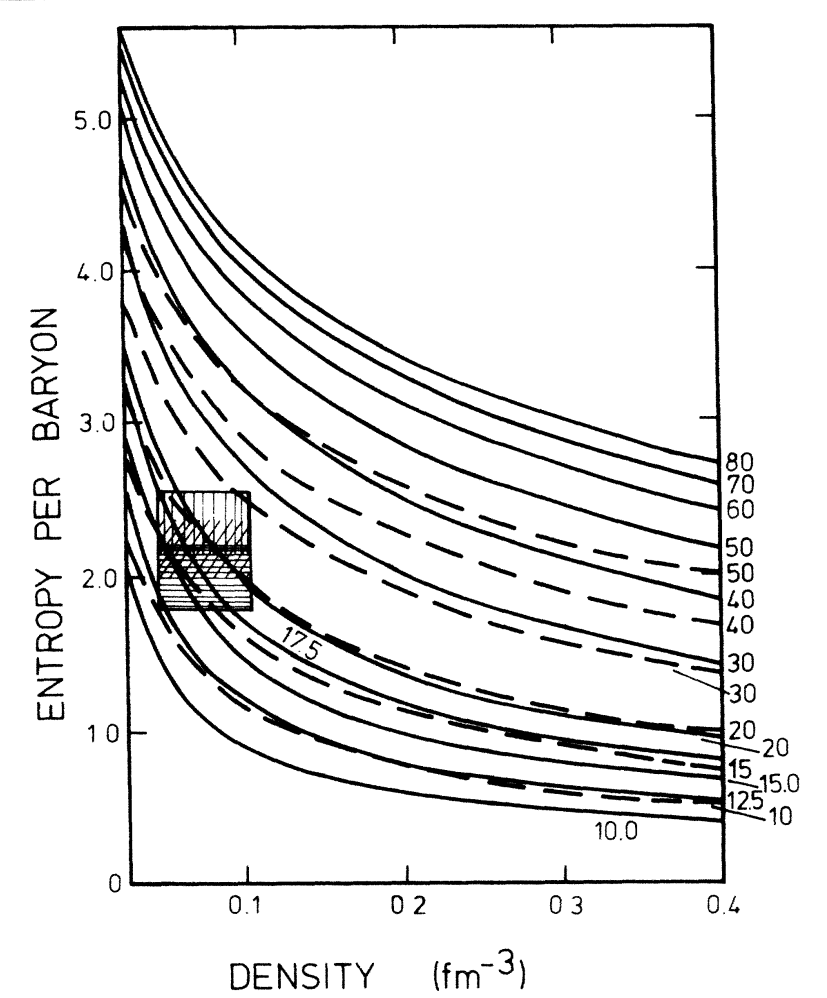

FIG. 1. Entropy per baryon (for $r=1$ ) as a function of density for different values of temperature (in megaelectronvolts). The shaded regions are the experimental observations of Ref. 2. Horizontal, inclined, and vertical lines are for temperatures $T=18,25$, and $35 \mathrm{MeV}$, respectively. The dashed curves represent the free-Fermi-gas entropy for an effective mass $m^{*}=0.7 \mathrm{~m}$. The numbers on the right give the temperature (in megaelectronvolts).

an effective mass $m^{*}=0.70 \mathrm{~m}$. We find that at low temperatures $(T \leq 30 \mathrm{MeV})$ the free Fermi gas with the above value of $m^{*}$ is a reasonable approximation to the results of our macroscopic calculation. It seems that all realistic calculations lead to this value of effective mass around the Fermi level and therefore to the same low-temperature entropy. In these calculations, the effective mass changes when the single-particle energy increases. This is the main reason for the deviation from the Fermi gas at high temperatures $(>30$ $\mathrm{MeV}$ ), as shown by Fig. 1 .

Figure 2 shows the importance of $m^{*}$ at various temperatures. Actually, the free-Fermi-gas entropy follows a scaling law. When the transformation $m^{*} \rightarrow \alpha m^{*}$, $T \rightarrow \alpha^{-1} T$ is applied (where $\alpha$ is a numerical constant), the entropy is conserved. The comparison in Fig. 2 demonstrates the importance of the interaction in the entropy production. It will be of interest to include this effect in the experimental analysis as well, although part of it is inherently built into the clusters automatically.

In conclusion, we have shown that fully self-

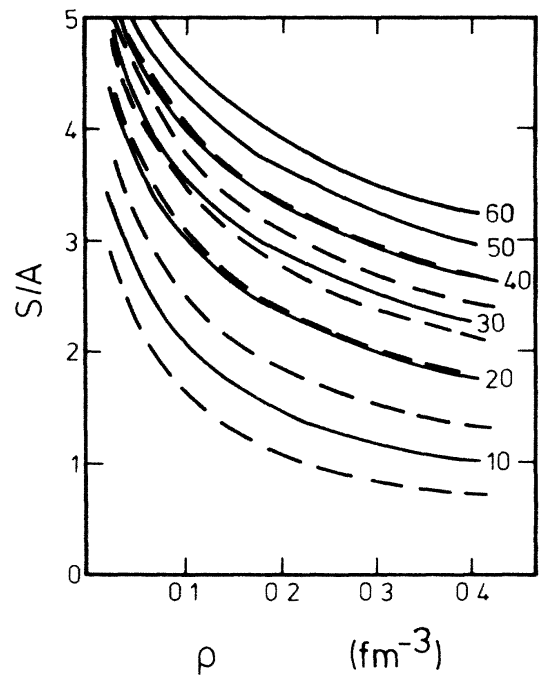

FIG. 2. Full curves: Entropy per baryon as a function of baryon density at different temperatures (indicated by the numbers, in megaelectronvolts) for the free Fermi gas with an effective mass of $m^{*}=1.5 m$ and with $r=1$. Dashed curves: Same for $m^{*}=m$.

consistent equation of state with realistic $N N$ interaction gives entropy in good agreement with the recent experimental data without our assuming the breakup into two phases. We also suggest that the free Fermi gas with effective mass is a potentially viable model in this context and deserves further investigations.

Thanks are due to C. Mahaux and A. Lejeune for enlightening discussions. One of the authors (R.K.T.) thanks C. Mahaux for the excellent hospitality at the University of Liège. This work was partially supported by Institut Interuniversitaire des Sciences Nucléaires (Belgium).

1P. J. Siemens and J. I. Kapusta, Phys. Rev. Lett. 43, 1486 (1979).

2B. V. Jacak et al., Phys. Rev. Lett. 51, 1846 (1983); B. V. Jacak, H. Stöcker, and G. Westfall, Phys. Rev. C 29, 1744 (1984)

${ }^{3}$ H. H. Gutbrod et al., Phys. Lett. 127B, 317 (1983); H. H. Gutbrod et al., Nucl. Phys. A400, 343c (1983).

${ }^{4}$ L. P. Csernai and J. I. Kapusta, Phys. Rep. 131, 223 (1986), and references therein.

${ }^{5}$ L. P. Csernai, Phys. Rev. Lett. 54, 639 (1985).

${ }^{6} \mathrm{G}$. Bertsch and J. Cugnon, Phys. Rev. C 24, 2514 (1981).

${ }^{7}$ H. Stöcker et al., Nucl. Phys. A400, 63c (1983).

${ }^{8}$ G. Fai and J. Randrup, Nucl. Phys. A381, 557 (1982).

9 J. Cugnon, in "Heavy Ion Collisions," edited by P. Bonche et al., NATO Advanced Studies Institute Series B, Vol. 130 (Plenum, New York, to be published).

${ }^{10}$ A. Lejeune, P. Grangé, M. Martzolff, and J. Cugnon, Nucl. Phys. (to be published).

${ }^{11}$ R. K. Tripathi, Phys. Lett. 101B, 369 (1981), and Phys. Rev. C 25, 1114 (1982). 
${ }^{12} \mathrm{C}$. Bloch and C. de Dominicis, Nucl. Phys. 10, 509 (1959).

13J.-P. Jeukenne, A. Lejeune, and C. Mahaux, Phys. Rep. 25C, 85 (1976).

${ }^{14} \mathrm{C}$. Mahaux, private communication.

15J. P. Elliott, A. D. Jackson, H. A. Mavromatis, E. A.
Sanderson, and B. Singh, Nucl. Phys. A121, 241 (1968);

N. I. Kassis, J. P. Elliott, and E. A. Sanderson, Nucl. Phys. A359, 386 (1981); R. K. Tripathi, J. P. Elliott, and E. A. Sanderson, Nucl. Phys. A380, 483 (1982).

16B. Friedman and V. R. Pandharipande, Nucl. Phys. A361, 502 (1981) 